\title{
Quasiparticle Trapping, Excitation, and Tunneling Times in Josephson Junctions with Spatially Inhomogeneous Electrodes
}

\author{
E.P.Houwman, A.A.Golubov, J.G.Gijsbertsen, J.Flokstra, \\ H.Rogalla, J.B.le Grand ${ }^{1}$, M.P.Bruijn ${ }^{1}$, and P.A.J. de Korte ${ }^{1}$, \\ University of Twente, P.O.B.217, 7500 AE Enschede, \\ The Netherlands, \\ ${ }^{1}$ SRON, Sorbonnelaan 2, 3584 CA Utrecht, The Netherlands.
}

\begin{abstract}
On the basis of a microscopic model of the proximity effect in an SS'-sandwich we have calculated effective trapping, excitation, and tunneling rates of the reduced gap region in an SS'IS"S junction as a function of temperature, voltage over the junction and strength of the proximity effect. We developed a simple model to describe the effect of magnetic fields on the gap of the superconductor, and calculated the trapping rate as function of the field. The experimentally determined gap reduction and quasiparticle loss times as function of the field were described in terms of this model.

PACS numbers: $74.40+k, 74.50+r$.
\end{abstract}

\section{INTRODUCTION}

For the development of junction $\mathrm{X}$-ray detectors there is a strong need for a quantitative model of the non-equilibrium superconductivity processes going on in the absorber after the X-ray photon impact. In a detector with a trapping layer, consisting of a proximity sandwich of two superconductors with different bulk energy-gaps, and used to enhance the quasiparticle (qp) collection and tunneling rate, ${ }^{1}$ there is an exchange of qps between the bulk of the absorber and the trap. This system may be described with two sets of Rothwarf-Taylor (RT)-equations, for the bulk and the trap respectively, coupled by an effective trapping time $\tau_{t r}$ and excitation time $\tau_{\text {exc }}$. Additionally an effective time constant $\tau_{\text {tun }}$ for tunneling out of the trap is needed in the RT-modeling.

The application of a magnetic field to junctions introduces also spatial modulation of the superconducting properties: the gap in the trapping layer will be reduced even more, changing all its time constants. An additional trapping-layer is created at e.g. the substrate-absorber interface, giving rise to qp loss.

In this paper we will give the results of a model from which the effective time constants are calculated as a function of temperature, voltage over the junction, and 
strength of the proximity effect. Secondly, the effect of a magnetic field is described in the low temperature limit. The field dependence of experimentally determined gap reductions and loss times are described with this model.

\section{THEORY}

A microscopic model of the proximity effect in a SS'-sandwich of dirty superconductors was developed for the case of a thin $S^{\prime}$-layer $\left(d \ll \xi_{S^{\prime}}\right)$ and a thick S-layer $\left(d_{S} \gg \xi_{S}\right) .{ }^{2}$ In the limit of good electrical contact between S and S', i.e. opposite to that of McMillan, where a finite potential barrier at the SS'-interface is assumed, the proximity effect can be described with two parameters, $\gamma_{m}=\left(\rho_{S} \xi_{S} / \rho_{S^{\prime}} \xi_{S^{\prime}}\right) /\left(d / \xi_{S^{\prime}}\right)$ and the critical temperature ratio $T_{c}^{\prime} / T_{c}$. The calculations in this paper are for a $\mathrm{Nb} / \mathrm{Al}$-sandwich with $T_{c}^{t} / T_{c}=0.14$, but a variation of the $T_{c}$-ratio for other metal combinations can be taken into account by a renormalization of $\gamma_{\mathrm{m}}{ }^{2}$

From this model we can calculate the normalized qp and pair densities of states $\bar{N}$ and $\bar{P}$, as a function of the distance $x$ from the SS'-interface and energy $\epsilon$. These functions enter into the spatial dependent expressions for the time constants for qp scattering with emission $\left(\tau_{e}\right)$, respectively absorption of a phonon $\left(\tau_{a}\right)$, and qp recombination $\left(\tau_{r}\right)$, which are extensions of the well-known Kaplan expressions for the spatially homogeneous superconductor. ${ }^{3,4}$

\subsection{Quasiparticle trapping and excitation in an SS'-sandwich}

The trapping time constant $\tau_{t r}$ of a single qp (with energy $\epsilon>\Delta_{0}, \Delta_{0}$ is the bulk gap in S) into the reduced gap region of an SS'-proximity sandwich, due to phonon emission, can now be defined $\mathrm{as}^{5}$

$$
\frac{1}{\tau_{t r}}=\frac{1}{N_{>}^{t o t}}\left|\frac{d N_{>}^{t o t}}{d t}\right|=\frac{1}{N_{>}}\left|\frac{d N_{>}}{d t}\right| \frac{1}{1+\alpha}+\frac{1}{N_{>}^{\prime}}\left|\frac{d N_{>}^{\prime}}{d t}\right| \frac{\alpha}{1+\alpha}, \alpha=\frac{N_{>}^{\prime}}{N_{>}}
$$

Here $N_{>}^{\text {tot }}, N_{>}$, and $N_{>}^{\prime}$ are the total number of qp with energy $\epsilon>\Delta_{0}$, respectively the qp numbers in $S$ and $S^{\prime}$. The trapping rate in $S$ is

$$
\left(\frac{1}{\tau_{\text {tr }}}\right)_{S}=\frac{1}{N_{>}}\left|\frac{d N_{>}}{d t}\right|=\int_{0}^{d_{S}} d x \int_{\Delta_{\mathbf{0}}}^{\infty} d \epsilon \frac{\bar{N}(\epsilon, x) f(\epsilon)}{\tau_{\varepsilon}(\epsilon, x)} / \int_{0}^{d_{S}} d x \int_{\Delta_{\mathbf{0}}}^{\infty} d \epsilon \bar{N}(\epsilon, x) f(\epsilon)
$$

and similarly for $\left(\tau_{t r}^{-1}\right)_{S^{\prime}}$. In the expression for $\tau_{e}^{-1}$ one takes only into account the scatter processes in which a phonon is emitted with energy $\Omega \geq\left(\epsilon-\Delta_{0}\right)$. Introducing an effective length $L_{e f f}$ of a trap with zero gap which gives the same total scattering rate as the reduced gap region in $\mathrm{S}$ by the relation $\left(\tau_{t r}^{-1}\right)_{S}=$ $\left(L_{e f f}(T) / d_{S}\right)\left(\tau_{e}^{-1}\left(\Delta_{g}=0\right)\right)=\left(L_{e f f} / d_{S}\right)\left(1.82 / \tau_{0}\right)$, and analogously a length $L_{e f f}^{\prime}$ for the S' layer, ${ }^{4,5}$ we obtain finally $(\alpha \ll 1)$,

$$
\frac{1}{\tau_{t r}}=\frac{1.82}{d_{s}}\left[\frac{L_{e f f}\left(T, \gamma_{m}\right)}{\tau_{0}}+\beta \frac{L_{e f f}^{\prime}\left(T, \gamma_{m}\right)}{\tau_{0}^{\prime}}\right]
$$

$\tau_{0}\left(\tau_{0}^{\prime}\right)$ are the material dependent time constants of the $S\left(S^{\prime}\right)$ metal, as defined by Kaplan. ${ }^{3}$ The factor $\beta=\alpha\left(d / d_{s}\right)$ is equal to $\left(v_{F} / v_{F^{\prime}}\right)\left(B^{\prime} / B\right)$, with $v_{F}\left(v_{F^{\prime}}\right)$ the Fermi- velocity of $S\left(S^{\prime}\right) . B^{\prime} / B$ is nearly equal 1 for $\gamma_{m} \ll 1$ and equal 

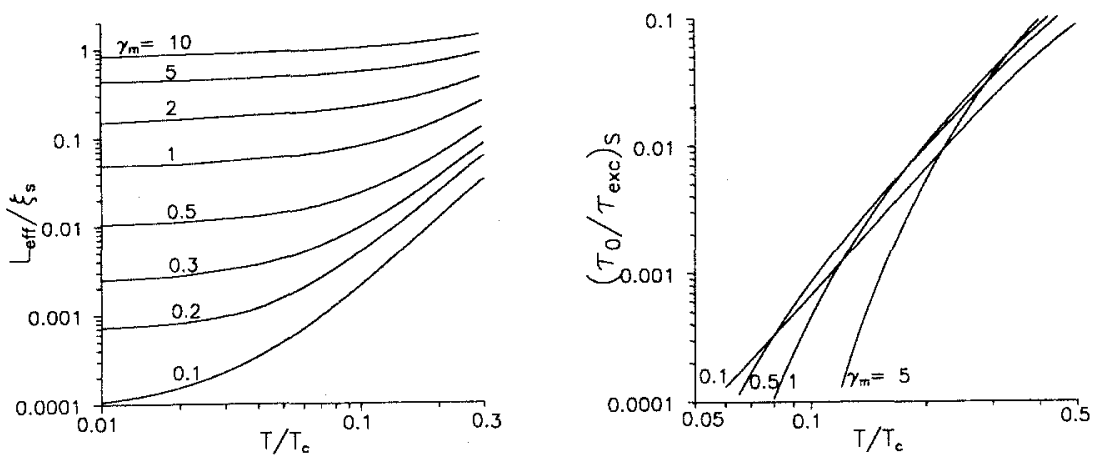

Fig. 1. (a) Effective trapping length $\left(L_{e f f}\right)$ and (b) excitation rate $\left(\tau_{0} / \tau_{e x c}\right)_{S}$ in $\mathrm{S}$ as function of temperature for various values of $\gamma_{m}$.

$\left(2 k_{B} T / \pi \Delta_{0}\right)^{1 / 2}$ for $\gamma_{m} \geq 1$ and low $T$. If the area $A$ of $\mathrm{S}$ is larger than the area $A^{\prime}$ of $\mathrm{S}^{\prime}, \tau_{t r}^{-1}$ is reduced with the factor $A^{\prime} / A$, assuming that the fraction of time the qp spend in the reduced gap region is proportional to $A^{\prime} / A$. In fig.1 $L_{e f f}$ is shown as function of temperature and strength of the proximity effect. For small $\gamma_{m}$ the effective length, and thus the trapping rate is very much temperature dependent, reaching a $T^{2}$-dependence at higher $T$. This reflects the increase of the number of high-energetic particles with higher $\tau_{e}^{-1}(\epsilon, x)$ when temperature is increased. The trapping rate increases with $\gamma_{m}$, since the trap becomes deeper. $L_{\text {eff }}^{\prime}$ shows nearly the same dependence on temperature and $\gamma_{m}$, but is normalized on $d$ instead of $\xi_{S}$.

A similar procedure is applied to derive an effective excitation rate out of the trap, resulting in ${ }^{5}$

$$
\frac{1}{\tau_{e x c}}=\left(\frac{\tau_{0}}{\tau_{e x c}}\right) s \frac{1}{1+\delta} \frac{1}{\tau_{0}}+\left(\frac{\tau_{0}^{\prime}}{\tau_{e x c}^{\prime}}\right) s^{\prime} \frac{\delta}{1+\delta} \frac{1}{\tau_{0}^{\prime}}
$$

with $\delta=N_{<}^{\prime} / N_{<}=\left(v_{F} / v_{F^{\prime}}\right)\left(E^{\prime} / E\right)$ the ratio of the number of qp in the trap in $\mathrm{S}^{\prime}$ and that in $\mathrm{S} . E^{\prime} / E$ is related to the effective tunneling length discussed further on, by $E^{\prime} / E=d / L_{e f f-t}^{*}\left(T, \gamma_{m}\right)$. In fig.1 the excitation rate $\left(\tau_{0} / \tau_{e x c}\right)_{S}$ in $S$ is shown. The rate in $S^{\prime}$ has a similar dependence on temperature and $\gamma_{m}$. The strong temperature dependence reflects the increasing number of high-energetic phonons that can excite the qp out of the trap.

\subsection{Tunneling rate in an SS'IS"S-junction}

The tunneling rate has to be considered separately for electron and hole-like qps, due to the different voltage dependence. Considering first the tunneling time $\tau_{\text {tun,12 }}^{e}$ of an electron-like qp out of the trap from electrode 1 to electrode 2 in an $\left(\mathrm{SS}^{\prime}\right)_{1} \mathrm{I}\left(\mathrm{S}^{\prime \prime} \mathrm{S}\right)_{2}$ junction. The rate $\left(\tau_{t u n, 12}^{e}\right)^{-1}=\left|d N_{<, 1}^{e} / d t\right| / N_{<, 1}^{e}$ can be expressed in terms of the tunneling current out of the trap $I_{<, 12}^{e}=e\left|d N_{<, 1}^{e} / d t\right|$, which depends on the densities of states at the S'I and IS" interfaces and the voltage $V$ over the junction. In the low temperature regime one can take the voltage dependent terms out of the tunneling integral and an effective tunneling length $L_{e f f-t}^{*}$ can be defined 

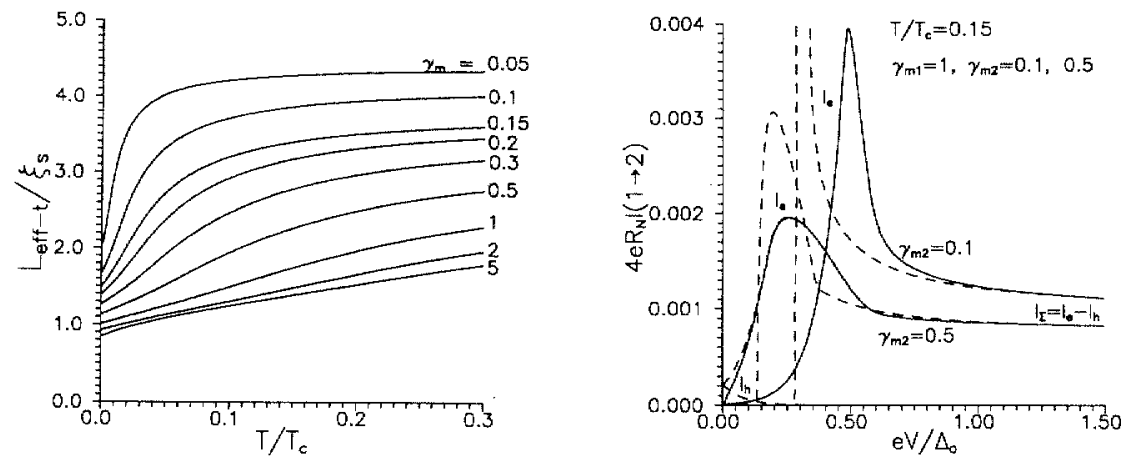

Fig. 2. (a) Effective tunneling length $\mathrm{L}_{\text {eff-t }}^{*}$ of the reduced gap region in an SS'-sandwich versus temperature for various $\gamma_{m}$-values. (b) $I_{<, 12}^{h}, I_{<, 12}^{e}$, and $I_{<, 12}^{\Sigma}$ versus biasvoltage, proportional to the tunneling rate $\left(\tau_{t u n, 12}^{e}\right)^{-1}$. The solid curves give the exact dependence, the dashed curves the approximate dependence through $\bar{N}_{2}$.

that is only dependent on temperature and $\gamma_{m} \cdot{ }^{5}$ With the area of the junction equal to the area $A^{\prime}$ of $\mathrm{S}^{\prime}$, the tunneling time becomes

$$
\tau_{t u n, 12}^{e}\left(V, T, \gamma_{m}\right)=4 e^{2} R_{N} A^{\prime} \frac{N_{1}(0) L_{e f f-t}^{*}\left(T, \gamma_{m}\right)+N_{1}^{\prime}(0) d}{\bar{N}_{2}\left(\Delta_{i g}+e V, x=-d\right)}
$$

Thus the voltage dependence of the tunnel time reflects the density of states $\bar{N}_{2}$ of the electrode to which the qps tunnel. In the spatially homogeneous case this expression reduces to that of Ivlev ${ }^{6}$ for the tunneling between homogeneous electrodes with different gaps and to the Ginsberg result for similar electrodes in the high voltage regime. ${ }^{7}$ It can be shown straightforwardly that the tunneling time for holes is related to that of the electron-like qps, by the relation $\tau_{\text {tun,12 }}^{h}(V, T)=\tau_{\text {tun,12 }}^{e}(-V, T)$. The total current of positive charge from electrode 1 to 2 is thus $I_{<, 12}^{\Sigma}=I_{<, 12}^{h}-I_{<, 12}^{e}$. In fig.2 the temperature dependence of $L_{t f f-t}^{*}$ is shown, as well as the voltage dependence of $\tau_{t u n}^{-1}$, through the currents $I_{<, 12}$, which reduces to the dependence through the term $\bar{N}_{2}\left(\Delta_{1 g}+e V, x=0\right)$ at low $\mathrm{T}$.

\subsection{Gap-reduction and quasiparticle loss by magnetic field}

For magnetic fields $H$ less than $H_{c 1}$ a dirty type II superconductor is in the Meissner state in which screening currents are induced in a surface layer with thickness $\lambda_{L}$. These currents act as a pairbreaking mechanism, giving rise to a reduction of the order parameter in the surface layer. The pairbreaking parameter is given $\mathrm{by}^{8}$

$$
\alpha_{p}=\frac{\gamma^{*}}{2} \Delta_{0}\left(H / H_{c}\right)^{2}
$$

with $\gamma^{*} \approx 1.78$ the Euler constant. The reduction of the gap in the density of states is then ${ }^{9}$

$$
\Delta_{g}(\zeta)=\Delta\left(1-\zeta^{2 / 3}\right)^{3 / 2}, \zeta<1
$$



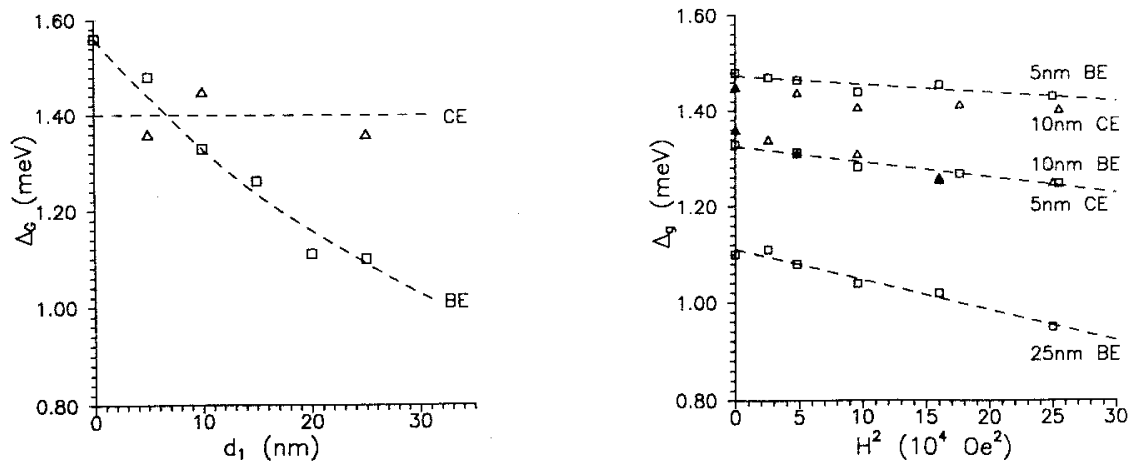

Fig. 3. (a) Measured energygap as function of Al-layer thickness $d_{1}$ at the BE ( $\square$ ), and at the CE with $d_{2}=3 \mathrm{~nm}(\triangle)$. The sloped curve gives the fit with the proximity theory; the horizontal line is a guide for the eye for $\Delta_{g 2}$. (b) Measured

gapvoltages of BE $(\square)$ and $\mathrm{CE}(\triangle)$ as function of applied field. The lines are guides for the eye for $\Delta_{g 1}$.

where $\zeta=\alpha_{p} / \Delta=\zeta_{0}\left(\Delta_{0} / \Delta\right)$ with $\zeta_{0}=\alpha_{p} / \Delta_{0}$. The reduction of the order parameter is given by

$$
\Delta=\Delta_{0} e^{-\pi \zeta / 4}
$$

For $\zeta \ll 1$ this gives a gap reduction which is nearly linear with $\zeta_{0}$ and thus quadratic in $H$. The trapping rate $\tau_{H}^{-1}$ in the surface layer at zero temperature was estimated as $^{8}$

$$
\frac{1}{\tau_{H}}=\frac{1.82}{\tau_{0}} \frac{L_{H}}{d_{s}}\left(2-A^{\prime} / A\right)
$$

where the effective trapping length is approximately $L_{H} \approx 1.3 \lambda_{L}\left(H / H_{c}\right)^{p}$ with $p \approx 10 / 3$.

\section{EXPERIMENTS}

The gapvoltages $\Delta_{g 1}$ and $\Delta_{g 2}$ of the base (BE) and counter (CE) electrodes of a series of $(\mathrm{Nb} / \mathrm{Al})_{1} / \mathrm{Alox} /(\mathrm{Al} / \mathrm{Nb} / \mathrm{Nb})_{2}$ tunnel junctions with $d_{1}=5,10,15,20$, and $25 \mathrm{~nm}$ (junctions 1 to 5 ) and $d_{2}=3 \mathrm{~nm}$ were determined, as a function of the applied magnetic field and at low temperatures. In fig. $3 \Delta_{g 1}$ versus $d_{1}$ is shown for $H=0$, together with the fitted theoretical curve, obtained from the proximity effect model. From the fit we have $\gamma_{m} / d_{1} \approx 0.032 \mathrm{~nm}^{-1} . \Delta_{g 2}$ is smaller and consequently the $\gamma_{m 2}$-value of the $\mathrm{CE}$ is larger than one should expect from the $\mathrm{Al}_{2}$-layer thickness. This is ascribed to degraded properties of the double $\mathrm{Nb}_{2}$-layer. Fig. 3 shows the field dependence of $\Delta_{g 1}(H)$ and $\Delta_{g 2}(H)$ for the junctions 1,2 , and 5 . It is seen that the gaps decrease with $H^{2}$ in accordance with eqs.(6-8). With increasing $d_{1}$ the gap $\Delta_{g 1}$ decreases more with $H$. This is ascribed to an decrease of $H_{c}$ with $d_{1}$, hence an increasing pairbreaking parameter $\alpha$, because the properties of the surface of the SS'-sandwich becomes more like those of $\mathrm{Al}$, which has a much smaller value of $H_{c}(\approx 100 O e)$ than $\mathrm{Nb}(\approx 2000 O e)$.

$\mathrm{X}$-ray measurements were performed on junctions for various fields. From the measured gapvoltages $\gamma_{m}$-values were determined from which the time-constants of 


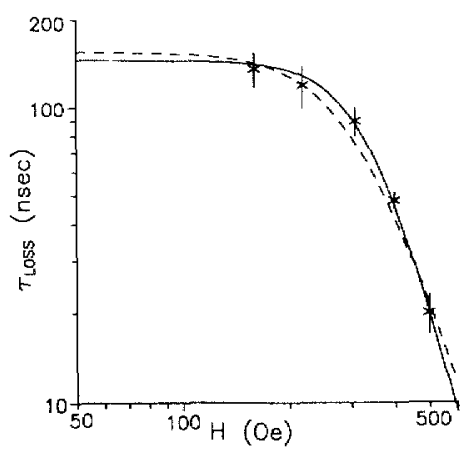

Fig. 4. Measured quasiparticle losstime $\tau_{\text {loss }}$ in the BE of junction $5\left(d_{1}=25 \mathrm{~nm}\right)$.

The solid (dashed) curve is a fit with $p=4.7(3.7)$.

the trap were estimated. ${ }^{10}$ With these values a loss-time constant $\tau_{\text {loss }}$ of the BE could be determined from the signal output of the junction. In fig. $4 \tau_{l o s s}$ versus $H$ is shown, together with two fits with the equation $\tau_{\text {loss }}^{-1}=\tau_{H}^{-1}(H, p)+\tau_{1}^{-1}$, with $\tau_{1}^{-1}$ some field independent loss rate, which dominates the loss process at low fields. From these fits we obtain $p \approx 3.7-4.7$ and $\tau_{1} \approx 150$ nsec. From the field dependence of $\tau_{\text {loss }}$ we conclude that this loss process is probably due to trapping of qps in reduced gap regions at the surface of the $\mathrm{BE}$, induced by the applied field. $\tau_{1}$ is ascribed to a degraded surface layer at the interfaces with substrate and insulation layers.

\section{ACKNOWLEDGEMENTS}

This work was funded by the Foundation for Fundamental Research of Matter.

\section{REFERENCES}

1. N.E.Booth, Appl. Phys. Lett. $\underline{50}, 293$ (1987)

2. A.A.Golubov et al., Proximity Effect in SS'IS"S Josephson Tunnel Junctions: Theory and Experiment, to be published

3. S.B.Kaplan et al., Phys. Rev. B14, 4854 (1976)

4. A.A.Golubov and E.P.Houwman, Physica C 205, 147 (1993)

5. A.A.Golubov et al., Quasiparticle and tunneling times in a Josephson junction with spatially inhomogeneous electrodes, submitted to Phys. Rev. B

6. B.Ivlev et al., Nucl. Instr. and Meth. A300, 127 (1991)

7. D.M.Ginsberg, Phys. Rev. Lett. $\underline{8}, 204$ (1962)

8. E.P.Houwman et al, Energy-gap Reduction and Quasiparticle Trapping due to Magnetic Fields, to be published

9. K.Maki, in Superconductivity Part 2, ed.:R.D.Parks, Marcel Dekker, Inc., New York (1969), p.1035

10. J.B.le Grand et al., Superconductive Junctions with Trapping Layers for the Detection of $X$-Rays, this proceedings 